Preprint 2007, Alfred Wegener Institute, Germany, M. Läuter, et al.

\title{
A parallel adaptive barotropic model of the atmosphere
}

\author{
Matthias LäUter $^{a}$, Dörthe HandorF ${ }^{a}$, NAtalja RAKOWskY ${ }^{b}$ \\ Jörn Behrens ${ }^{c}$, Stephan Frickenhaus ${ }^{c}$, Meike Best ${ }^{c}$ \\ KLaus DethlofF ${ }^{a}$, Wolfgang HilleR ${ }^{c}$ \\ ${ }^{a}$ Alfred Wegener Institute for Polar and Marine Research, Potsdam, Germany \\ ${ }^{b}$ Technische Universität Hamburg-Harburg, Computing Center, Hamburg, Germany \\ ${ }^{c}$ Alfred Wegener Institute for Polar and Marine Research, Bremerhaven, Germany
}

March 19, 2007

\begin{abstract}
The parallel adaptive model PLASMA has been developed for modeling a barotropic atmosphere. This model adapts the computational grid at every time step according to a physical error indicator. Thus, compared to uniform grid experiments the number of grid points is reduced significantly. At the same time, the error increases only slightly, when comparing with uniform grid solutions.

For the discretization of the underlying spherical shallow water equations a LagrangeGalerkin method is used. The unstructured triangular grid is maintained by the grid generator amatos and the large linear systems are solved by the parallel solver interface FoSSI. Experimental convergence is shown by means of steady-state and unsteady analytical solutions. PLASMA yields satisfactory results for quasi standard experiments, that is the Rossby-Haurwitz wave and zonal flows over an isolated mountain.
\end{abstract}

Key words: Atmospheric flow, Shallow water equations, Adaptive grid, Parallelization, Analytical test cases

MSC 35Q30, 65Z05, 76N15

\section{Introduction}

Atmospheric flows are largely determined by nonlinear interactions between processes at widely varying spatial and temporal scales. Thus, the realistic representation of these nonlinear multi-scale interactions is important for climate modeling as well as for numerical weather prediction. Considering the large-scale circulation, the interaction between zonal flow and planetary Rossby waves plays a crucial role for climate variability on time-scales from seasons to decades. To study these and other multi-scale interactions, the application of a global atmospheric model with a flow-dependent, i.e. dynamically adaptive grid may be appropriate.

The purpose of this paper is to introduce the new, adaptive global atmospheric model PLASMA (Parallel LArge-scale Self-adaptive Model of the Atmosphere). Within

Published in J. Comput. Phys. (2007), 223(2), 609-628 
Preprint 2007, Alfred Wegener Institute, Germany, M. Läuter, et al.

PLASMA, the model equations are solved on an unstructured, triangular grid, which is dynamically adapted to the evolving atmospheric flow every time step. The dynamic grid adaptation allows spatial resolution to increase locally over regions of interest within the whole model domain, thus keeping the computational costs limited.

Nowadays, atmospheric models with fixed, uniform horizontal resolution are most common. These models can resolve nonlinear multi-scale interactions only over a very limited range of spatial scales. To overcome this limitation, several approaches for a locally increased spatial resolution over areas of interest have been developed especially for atmospheric regional models (see also [18] for an overview). The most important approaches are nested grids, stretched grids and dynamically adapted grids.

Nested modeling systems have been developed since the 1970s for numerical weather prediction, e. g. [12], and later on for regional climate modeling, e. g. [22]. The nesting technique consists in embedding a high-resolution regional model into a lowresolution model, where the latter provides the time-dependent boundary forcing for the high-resolution model. The nesting technique can be either one-way, see [12], or two-way interactive, see [50, 34]. In simpler one-way nesting, large-scale information is given to the regional model, but no feedback from the high-resolution model to the low-resolution nesting model is possible. Two-way nesting includes feed back from the regional domain to the large scales, thus reducing potential mismatch between the high-resolution model and the low-resolution model.

An alternative technique for variable-resolution models is the stretched grid approach. Pioniering work on this approach for grid point models has been done by Staniforth and Mitchell in [46]. By stretching grid intervals outside a fixed, uniform fine-resolution area of interest uniformly over the rest of the globe, a single global variable-resolution grid is obtained. The main advantage of this approach is that it includes the two-way feedback between large-scale and regional-scale circulation. Stretched-grid regional climate simulations are becoming more common since the 1990s (see the studies $[13,18,35]$ ) and leading to the international SGMIP (Stretched-Grid Model Intercomparison Project) initiative, see [17]. For spectral models, a stretched grid can be obtained by applying a stretching coordinate transformation, see [42]. Several global atmospheric models are based on this approach, together with a rotation of the poles, see $[11,25]$.

The stretched grid approach with one fixed area of interest has been further developed in [19] to a grid design with multiple areas of interests which allows the simulation of different regional climates simultaneously. Even more flexibility with respect to areas and features of interest can be gained with dynamically stretched grids based on time-dependent global coordinate transformations. By redistributing a constant number of grid points a dynamically adapted moving grid is obtained. Probably the first atmospheric application of this method was developed by Dietachmayer and Droegemeier [14] solving the one-dimensional viscous Burger's equation. One- and two-dimensional adaptive advection problems have been treated in [27] whereas in [39] a 3D anelastic, non-hydrostatic model based on this time-dependent 
Preprint 2007, Alfred Wegener Institute, Germany, M. Läuter, et al.

coordinate transformation has been developed.

Instead of moving the grid with time, another dynamic grid adaptation technique inserts or removes grid points depending on a feature or process of interest. The first atmospheric model using this technique, a 3D limited-area model, has been introduced in [44] and [43]. More recently Bacon et al. [2] have developed the operational Multiscale Environmental Model with Grid Adaptivity (OMEGA), which is an adaptive non-hydrostatic regional weather and dispersion model.

On the way to the development of 3D global dynamically adaptive models, several spherical adaptive 2D shallow water models have been developed. The spherical shallow water equations comprise the essential physical phenomena that are included in the full set of primitive equations. Thus, they provide a test environment for the horizontal discretization methods and the adaptive grid refinement before going to the development of full 3D models.

Statically adaptive shallow water models on the sphere have been introduced in [41], [16] and [5]. Until now, spherical dynamically adaptive shallow water models are not wide-spread. Recently, Jablonowski proposed in [28, 29] an adaptive grid refinement technique for the hydrostatic Lin-Rood dynamics package based on a conservative and monotonic finite volume discretization in flux form. The corresponding dynamical core on the sphere has been run in a $2 \mathrm{D}$ shallow water model configuration as well as in a full 3D hydrostatic configuration. The proposed grid refinement technique has been developed for a quadrilateral latitudinal-longitudinal grid using a block-structured data-layout.

Here, we have developed a different dynamically adaptive shallow-water model, the model PLASMA. The requirements for underlying grid refinement strategy had been: (i) high flexibility in providing areas with high and low resolution, (ii) no abrupt changes in grid resolution, (iii) grid refinement depending on a feature or process of interest, (iv) automated refinement and coarsening of the grid. Furthermore, we have to demand stability of the numerical method for different spatial resolutions.

To fullfill these requirements, we approximate the sphere by a polyhedron consisting in an adaptive, unstructered triangular grid with the nodes situated on the sphere. The grid is generated by the mesh generator amatos, see [7]. Its refinement strategy is controlled by the atmospheric flow processes. A Lagrange-Galerkin method is used to discretize the governing equations. The Lagrange-Galerkin method is a combination of the semi-Lagrangian method for the temporal and the finite element method for the spatial discretization. These discretization methods lead to large linear systems of equations which are solved by means of parallel linear solver interfaces, provided by the package FoSSI, see [20].

The governing continuous equations of PLASMA are described in section 2, whereas their numerical discretization by a Lagrange-Galerkin method is given in section 3 . The dynamically adaptive grid generation technique is presented in section 4 followed by the introduction of the parallel linear solver interfaces in section 5 . In section 6, PLASMA is validated with special emphasis on the study of the convergence 
Preprint 2007, Alfred Wegener Institute, Germany, M. Läuter, et al.

properties. This is achieved by means of several known shallow water test cases, including steady analytical and non-analytical cases, see [49]. Furthermore, recently introduced unsteady analytical test cases in [33] are applied. In order to demonstrate the ability of PLASMA to simulate the most important feature of the extratropical large-scale circulation, planetary Rossby-waves, numerical experiments are presented in section 6 as well. The paper concludes with a summary.

\section{Spherical shallow water equations}

PLASMA is based on the spherical shallow water equations. These barotropic equations are derived from the non-divergent primitive equations in $\mathbb{R}^{3}$ for a homogeneous atmosphere with small vertical velocity components, see e.g. [24, 37, 31]. In PLASMA the spherical shallow water equations are used in a scalar formulation in terms of the prognostic variables vorticity, divergence and geopotential.

At first, some notation is introduced, the constants $a=6.371221 \cdot 10^{6} \mathrm{~m}$ for the Earth radius, $\Omega=7.292 \cdot 10^{-5} 1 / \mathrm{s}$ for the Earth's angular velocity and $g=9.81 \mathrm{~m} / \mathrm{s}^{2}$ for the Earth's acceleration due to gravity. Further, the equations are considered in a time interval $(0, T) \subset \mathbb{R}$ and in the spatial domain $S=\left\{\mathbf{x} \in \mathbb{R}^{3}|| \mathbf{x} \mid=a\right\}$. For two vectors $\mathbf{x}, \mathbf{y} \in \mathbb{R}^{3}$ the scalar product is denoted by $\mathbf{x} \cdot \mathbf{y}$.

The advective formulation of the spherical shallow water equations in cartesian coordinates, known from e.g. [10, 49, 23, 9], can be written as

$$
\begin{aligned}
\frac{d \mathbf{u}}{d t}+\nabla_{S} \Phi & =-f \mathbf{k} \times \mathbf{u}-\frac{|\mathbf{u}|^{2}}{|\mathbf{x}|} \mathbf{k}, \\
\frac{d\left(\Phi-\Phi_{B}\right)}{d t}+\left(\Phi-\Phi_{B}\right) \operatorname{div}_{S} \mathbf{u} & =0 \\
\mathbf{u} \cdot \mathbf{k} & =0 .
\end{aligned}
$$

Here, $\mathbf{u}(\mathbf{x}, t) \in \mathbb{R}^{3}$ and $\Phi(\mathbf{x}, t) \in \mathbb{R}$ are the wind field and the geopotential height field, $\mathbf{k}(\mathbf{x})=\frac{\mathbf{x}}{|\mathbf{x}|}$ is the normal vector in vertical direction, $f=2 \Omega(0,0,1) \cdot \mathbf{k}$ is the Coriolis parameter and $\Phi_{B}(\mathbf{x}) \in \mathbb{R}$ is the given geopotential height field of the Earth's orography. The material derivative of a function $h$ is defined by $\frac{d h}{d t}=$ $\partial_{t} h+\mathbf{u} \cdot \nabla_{S} h$, the horizontal gradient by $\nabla_{S} h=\nabla h-(\mathbf{k} \cdot \nabla h) \mathbf{k}$, the horizontal divergence of a vector field $\mathbf{v}$ by $\operatorname{div}_{S} \mathbf{v}=\operatorname{div}(\mathbf{v}-\mathbf{v} \cdot \mathbf{k} \mathbf{k})$ and the horizontal Laplacian by $\Delta_{S} h=\operatorname{div}_{S} \nabla_{S} h$. System (1) is equivalent to the scalar formulation of the spherical shallow water equations which consists of the prognostic equations

$$
\begin{aligned}
\frac{d \zeta}{d t}+\zeta \delta+f \delta & =-\mathbf{u} \cdot \nabla_{S} f \\
\frac{d \delta}{d t}+\Delta_{S} \Phi-f \zeta & =-(\mathbf{k} \times \mathbf{u}) \cdot \nabla_{S} f-J(\mathbf{u}) \\
\frac{d\left(\Phi-\Phi_{B}\right)}{d t}+\left(\Phi-\Phi_{B}\right) \delta & =0
\end{aligned}
$$


Preprint 2007, Alfred Wegener Institute, Germany, M. Läuter, et al.

and the Helmholtz decomposition

$$
\begin{aligned}
-\Delta_{S} \psi & =\zeta, \\
\Delta_{S} \chi & =\delta, \\
\operatorname{rot}_{S} \psi+\nabla_{S} \chi & =\mathbf{u} .
\end{aligned}
$$

The prognostic variables are vorticity, divergence and the geopotential field denoted by $\zeta(\mathbf{x}, t), \delta(\mathbf{x}, t), \Phi(\mathbf{x}, t) \in \mathbb{R}$, respectively. The diagnostic variables are the wind field $\mathbf{u}$, the stream function $\psi(\mathbf{x}, t) \in \mathbb{R}$ and the velocity potential $\chi(\mathbf{x}, t) \in \mathbb{R}$. The functional $J$ is defined by $J(\mathbf{u})=\sum_{i, j=1}^{3}\left(\nabla_{S} u_{i}\right)_{j}\left(\nabla_{S} u_{j}\right)_{i}+\mathbf{u} \cdot \mathbf{u}$.

\section{Lagrange-Galerkin method}

The model PLASMA computes the numerical solution of a viscous version of system (2), (3). Because viscosity vanishes asymptotically, this solution can be interpreted as a vanishing viscosity limit of system (2), (3). For that purpose, the LagrangeGalerkin method, e.g. see [47], is applied to the viscous spherical shallow water equations. The Lagrange-Galerkin method is a combination of the semi-Lagrangian method for the temporal and the finite element method for the spatial discretization on the sphere. Grid adaptation is accomplished in every time step. Based on a physical error indicator, in the time step $n$ a new triangulation $\mathcal{T}^{n+1}$ of $S$ is created with the grid generator in section 4 . A linear system of equations for the prognostic variables at time $t^{n+1}$ is constructed with the finite element method on $\mathcal{T}^{n+1}$. In doing so, on the right hand side the semi-Lagrangian method leads to trajectory computations and interpolations of variables at time step $n$ on the triangulation $\mathcal{T}^{n}$.

\subsection{Artificial viscosity}

Numerical modeling of advection dominated processes has to deal with energy transport from larger to smaller physical wave lengths. Because the computational grid gives a lower limit for the discrete wave lengths an energy accumulation in the small scales can lead to numerical instabilities, e. g. see [36]. One possiblity to circumvent this phenomenon is damping with artificial viscosity.

Due to these reasons, for the application of the Lagrange-Galerkin method the viscous spherical shallow water equations are considered. They consist of the system (2), (3) and the additional artificial viscosity terms $-\nu \Delta_{S} \zeta,-\nu \Delta_{S} \delta$ and $-\nu \Delta_{S} \Phi$ on the left hand sides of the equations in system (2), respectively. In doing so, the space dependent viscosity parameter $\nu(\mathbf{x}, t) \in \mathbb{R}_{\geq 0}$ is chosen proportional to the local grid resolution $\Delta x$. Thus, the asymptotic limit of the discrete viscous spherical shallow water equations yields a numerical solution of system (2). 
Preprint 2007, Alfred Wegener Institute, Germany, M. Läuter, et al.

\subsection{Semi-Lagrangian method}

The semi-Lagrangian method is an appoximation of the material derivative $\frac{d h}{d t}$ of a scalar function $h$, see [45]. Within one time step, each grid point $\mathbf{x}$ is treated as a Lagrangian particle and $\frac{d h}{d t}$ at the point $\mathbf{x}$ is approximated by a difference quotient along the trajectory of the point. In PLASMA, trajectories are computed by a first order explicit Euler method which leads to a first order approximation of the material derivatives.

Let us consider a function $h(\mathbf{x}, t) \in \mathbb{R}$ within the time step $t^{n} \rightarrow t^{n+1}$. The material derivative $\frac{d h}{d t}$ is derived on each grid point $\mathbf{x} \in S$ implicitly at time $t^{n+1}$. Therefore, the trajectory function $\mathbf{X}$ is considered starting at $\mathbf{x}$ fulfilling the equation

$$
\partial_{\tau} \mathbf{X}\left(\mathbf{x}, t^{n+1} ; \tau\right)=\mathbf{u}\left(\mathbf{X}\left(\mathbf{x}, t^{n+1} ; \tau\right), \tau\right), \quad \mathbf{X}\left(\mathbf{x}, t^{n+1} ; t^{n+1}\right)=\mathbf{x}
$$

for $\tau \in\left[t^{n}, t^{n+1}\right]$. This system is solved with the time extrapolated value $\mathbf{u}\left(\mathbf{x}, t^{n+1}\right)$ by an explicit Euler method yielding the numerical solution $\mathbf{X}^{*}(\mathbf{x})=\mathbf{X}^{*}\left(\mathbf{x}, t^{n+1} ; t^{n}\right)=\mathbf{X}\left(\mathbf{x}, t^{n+1} ; t^{n}\right)+O\left(\Delta t^{2}\right) . \quad$ Using $\frac{d h}{d t}\left(\mathbf{x}, t^{n+1}\right)=$ $\frac{h\left(\mathbf{x}, t^{n+1}\right)-h\left(\mathbf{X}\left(\mathbf{x}, t^{n+1} ; t^{n}\right), t^{n}\right)}{\Delta t}+O(\Delta t)$, we obtain the first order approximation of the material derivative at the point $\mathbf{X}^{*}$ for a smooth (Lipschitz continuous) $h$

$$
\frac{d h}{d t}\left(\mathbf{x}, t^{n+1}\right)=\frac{h\left(\mathbf{x}, t^{n+1}\right)-h\left(\mathbf{X}^{*}(\mathbf{x}), t^{n}\right)}{\Delta t}+O(\Delta t) .
$$

\subsection{Finite element method on the sphere}

The starting point for the finite element method is the weak formulation of system (2), (3) at time $t^{n+1}$. For the clarity of presentation, in this section we confine ourselves to the treatment of the vorticity equation in (2). Let us denote $h^{n+1}(\mathbf{x})=$ $h\left(\mathbf{x}, t^{n+1}\right)$ for a function $h(\mathbf{x}, t)$. Then, the weak formulation of the vorticity equation is given by

$$
\begin{aligned}
\int_{S}\left({\frac{d \zeta^{n+1}}{d t}}^{\left.n+\left(\zeta^{n+1}+f\right) \delta^{n+1}\right)}\right. & \varphi d \sigma \\
& +\int_{S} \nu \nabla_{S} \zeta^{n+1} \cdot \nabla_{S} \varphi d \sigma=\int_{S}\left(-\mathbf{u}^{n+1} \cdot \nabla_{S} f\right) \varphi d \sigma
\end{aligned}
$$

for arbitrary test functions $\varphi(\mathbf{x}) \in \mathbb{R}$. With the approximations

$$
\zeta^{n+1} \delta^{n+1}=\frac{1}{2}\left(\zeta^{n+1} \delta^{n}+\zeta^{n} \delta^{n+1}\right)+O(\Delta t), \quad \mathbf{u}^{n+1}=\mathbf{u}^{n}+O(\Delta t)
$$


Preprint 2007, Alfred Wegener Institute, Germany, M. Läuter, et al.

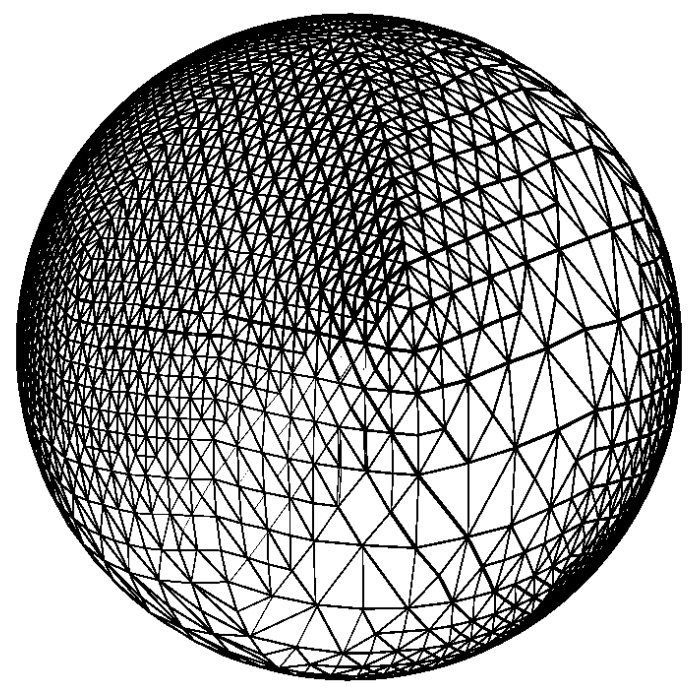

Figure 1: Triangulation $\mathcal{T}^{n+1}$ on $S$ at time $t^{n+1}$.

and the semi-Lagrangian time derivative in Eq. (4) one obtains the linearized semidiscrete formulation of the vorticity equation

$$
\begin{aligned}
\int_{S}\left(\zeta^{n+1}(1+\right. & \left.\left.\frac{\Delta t \delta^{n}}{2}\right)+\delta^{n+1} \Delta t\left(f+\frac{\zeta^{n}}{2}\right)\right) \varphi d \sigma \\
& +\Delta t \int_{S} \nu \nabla_{S} \zeta^{n+1} \cdot \nabla_{S} \varphi d \sigma=\int_{S}\left(\zeta^{n}\left(\mathbf{X}^{*}\right)-\Delta t \mathbf{u}^{n} \cdot \nabla_{S} f\right) \varphi d \sigma .
\end{aligned}
$$

At every time step the grid is adapted to the dynamical model situation. Therefore, a physical error indicator is derived for model variables at time $t^{n}$, see section 4.1. Based on this error the triangulation $\mathcal{T}^{n+1}$ with $N$ points is created on $S$ for the new time $t^{n+1}$, see Fig. 1 and section 4.2. Piecewise linear basis functions $\left(\varphi_{i}\right)_{i=1, . ., N}$ with respect to $\mathcal{T}^{n+1}$ are considered and the approach

$$
\zeta^{n+1}(\mathbf{x})=\sum_{i=1}^{N} \zeta_{i}^{n+1} \varphi_{i}(\mathbf{x}), \quad \delta^{n+1}(\mathbf{x})=\sum_{i=1}^{N} \delta_{i}^{n+1} \varphi_{i}(\mathbf{x})
$$

is chosen for the model variables. The semi-Lagrangian method leads to the evaluation of the upwind points $\mathbf{X}^{*}$ and of $\zeta^{n}\left(\mathbf{X}^{*}\right)$ in Eq. (5). Because $\zeta^{n}$ is piecewise linear on $S$, this function evaluation can be taken as a linear interpolation of $\zeta^{n}$. Thus, Eq. (5) yields a linear system of equations for the unknown coefficients $\left(\zeta_{i}^{n+1}\right)_{i=1, . ., N}$ and $\left(\delta_{i}^{n+1}\right)_{i=1, . ., N}$, which is solved at every time step, see section 5 . Numerical experiments have yielded the heuristic perception, that stability as well as accuracy properties are much better with a piecewise quadratic choice for the geopotential $\Phi^{n+1}$. 


\section{Adaptive grid generation}

Knowing the model variables at the time $t^{n}$ on the triangulation $\mathcal{T}^{n}$ of $S$ an adapted triangulation $\mathcal{T}^{n+1}$ is created. Therefore, a physical error indicator is employed to mark individual elements of $\mathcal{T}^{n}$ for refinement and coarsening, respectively. The grid generator amatos uses these marks to modify $\mathcal{T}^{n}$ and to create the adapted triangulation $\mathcal{T}^{n+1}$ for the time $t^{n+1}$.

\subsection{Physical error indicator}

Let the triangulation $\mathcal{T}^{n}$ be the set of all elements of the grid on $S$ at time $t^{n}$. An error estimator $\eta$ of the numerical solution $\zeta, \delta, \Phi$ of the system $(2),(3)$ is a function, which assigns a scalar value $\eta(T)$ to every grid element $T \in \mathcal{T}^{n} . \eta(T)$ represents the quality of $\zeta, \delta, \Phi$ in $T$. Until now there is no rigorous mathematical error estimator for the spherical shallow water equations. That is why the physical error indicator

$$
\eta(T)=\left(\int_{T} \zeta^{2}+\delta^{2} d \sigma\right)^{\frac{1}{2}}, \text { for } T \in \mathcal{T}^{n}
$$

is introduced resulting in the corresponding global error indicator $\eta_{g}=$ $\left(\sum_{T \in \mathcal{T}^{n}} \eta(T)^{2}\right)^{\frac{1}{2}}$. This choice of $\eta(T)$ is based on the perception, that high flow gradients will lead to high discretization errors. $\eta$ is uniformly distributed, if there is a constant value $\eta_{0}$ that the relation $\eta(T)=\eta_{0}$ holds for all $T \in \mathcal{T}^{n}$. Thereby, $\eta_{0}$ can be expressed by $\eta_{0}=\frac{\eta_{g}}{\sqrt{N_{e}}}$, with the number of grid elements $N_{e}$. The triangulation $\mathcal{T}^{n+1}$ for the future time $t^{n+1}$ is constructed with the aim, that $\eta$ is nearly uniformly distributed. If $\eta$ is already uniformly distributed, the triangulation remains unmodified, that is $\mathcal{T}^{n+1}=\mathcal{T}^{n}$. If $\eta$ is not uniformly distributed, a tolerance interval $0<\underline{\eta}<\eta_{g}<\bar{\eta}$ is fixed. Due to experimental results, the values $\underline{\eta}=\frac{4}{5} \eta_{g}$ and $\bar{\eta}=\frac{6}{5} \eta_{g}$ are chosen for the model. Then, the sets

$$
\mathcal{F}:=\left\{T \in \mathcal{T}^{n} \mid \eta(T)>\frac{\bar{\eta}}{\sqrt{N}_{e}}\right\}, \quad \mathcal{C}:=\left\{T \in \mathcal{T}^{n} \mid \eta(T)<\frac{\underline{\eta}}{\sqrt{N}_{e}}\right\}
$$

are defined which contain the elements that differ too strongly from the mean value. In $\mathcal{T}^{n}$ the members of the subsets $\mathcal{F}$ and $\mathcal{C}$ are now marked for refinement and coarsening, respectively.

\subsection{Grid generation}

Triangular adaptive grid generation involves an unstructured problem. Therefore, advanced techniques for refinement, data structures and data handling are required. These techniques are implemented in the mesh generation library amatos (Adaptive Mesh generator for Atmospheric and Oceanic Simulation) [6]. The main paradigm 


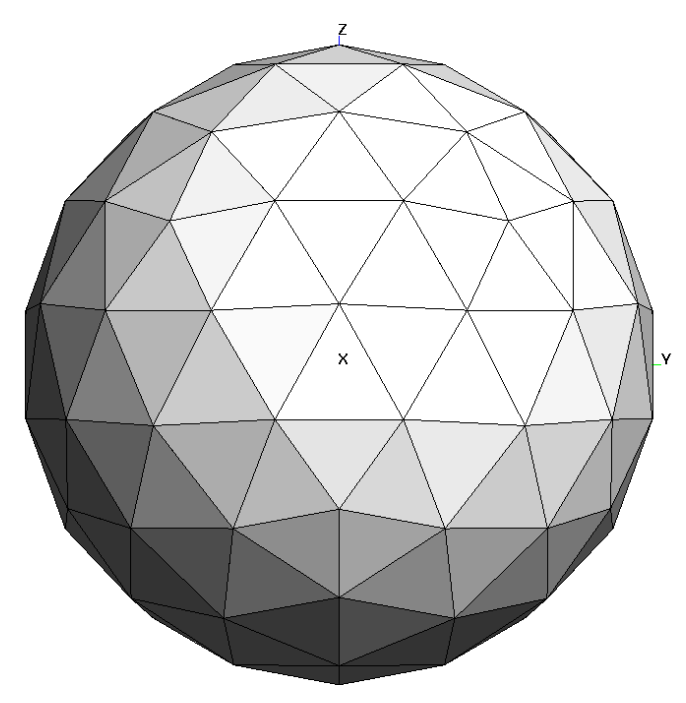

Figure 2: The initial mesh based on a bucky ball triangulation.

underlying amatos' data organization is the strict separation of (mainly integer) grid generation related operations and (mainly floating point) numerical operations. A gather/scatter step has to take place prior to data processing. The main advantage of this small overhead is that numerical operations can be performed on consecutive data structures, facilitating low level and automatic compiler optimization.

The grid generation part comprises an object oriented data management structure. The mesh consists of mesh atoms: nodes, edges and cells. Edges have knowledge of their neighbors, providing the main connectivity information. Mesh refinement is achieved by a bisection-of-marked-edge strategy [40,4]. This refinement strategy leads to conforming meshes (i.e. meshes without hanging nodes) and yields a favourable lower bound for the smallest interior angles of cells. Furthermore, this strategy is very simple from the algorithmic point of view, since it does not need to track exceptional refinements, and is well suited for the generation of space-filling curve orderings (see below). Finally, coarsening a locally refined mesh is easily achieved. A binary tree data structure maintains locally refined meshes efficiently. Especially, element search operations are accelerated to $\mathcal{O}(\log m)$ operational complexity, where $m$ is the number of mesh cells.

When using amatos, a coarse initial mesh has to be specified. Two parameters control the mesh refinement: a fine level defines a mesh level of uniform refinement, while a coarse level defines the minimum level of refinement (i.e. the lowest permitted resolution). The local degree of grid refinement is controlled by an iterative refinement or coarsening according to the sets $\mathcal{F}$ and $\mathcal{C}$. amatos is capable of handling plane and spherical two-dimensional meshes. In spherical geometries, newly inserted mesh nodes are projected to the sphere's surface by central projection. An initial mesh based on a bucky ball triangulation [26] is shown in Fig. 2.

An important problem with unstructured meshes on high performance computing devices is the mesh partitioning. amatos uses a space-filling curve (SFC) approach 
Preprint 2007, Alfred Wegener Institute, Germany, M. Läuter, et al.

to find a mesh partitioning. The SFC resembles the refinement process and needs only one bit shift per refined element [7]. This type of SFC preserves neighborhood relations and yields connected domains. Perfect load balancing is achieved with a small increase in the edge cut compared to other partitioning techniques [8].

amatos supports finite element calculations by a flexible run-time management of arbitrary element types. Unknowns in elements are allowed to be located on nodes, edges and cell interiors of the mesh (see section 3.3). The unknowns or degrees of freedom are sorted by the SFC in consecutive order. This leads to matrix bandwidth reduction and significant improvement of convergence in preconditioned CG-like methods [7].

\section{Linear equations solver interface}

Choosing an appropriate linear solver for a highly complex finite element model is a challenging task. Especially with growing problem complexity during the development of the model, formerly successful algorithms may turn out to be no longer applicable. Therefore, a variety of powerful solvers is needed that can be easily exchanged. The linear equations solver applied in PLASMA is attached via the Family of Simplified Solver Interfaces (FoSSI, see [20]) which provides a very simple user interface to MPI-parallel iterative solvers from PETSc, hypre, PILUT, AZTEC, see $[3,15,30,48]$, and even to the direct solver MUMPS, see [1].

FoSSI makes accessible a variety of parallel algorithms and methods such as Krylov subspace iterators like CG, GMRES, BiCGStab preconditioned by domain decomposition (PETSc, AZTEC, hypre), multilevel incomplete factorization (PILUT) or algebraic multigrid (hypre) and parallel multifrontal direct methods (MUMPS). These are frequently tested, state-of-the-art parallel libraries, offering superior performance and providing efficient memory usage. It is noteworthy, that libraries such as PETSc and hypre also offer several methods to select solvers, even from external solver libraries. However, compared to FoSSI there are still many limitations in the flexibility, e.g. concerning different interfaces and data structures in different libraries.

FoSSI is implemented as a collection of interface routines to the different solver libraries. By this, it is possible to modify each interface independently from the others, e.g., for using new solver features or upgrading to a new solver library release with syntax changes. All FoSSI-interfaces consist of only one routine with a task specifying parameter, allowing the selection of several operations and configuration options in one call. Furthermore, the same interface may be called several times for different phases of the problem solution such as matrix structure setup, matrix value feed, factorization, solution and clean-up. Many linear problems may be kept in memory simultaneously. As it is even possible to define a separate MPI-communicator on each problem, several linear problems can be treated in parallel.

Benefiting from the flexiblity of FoSSI a couple of linear solvers have been tested within PLASMA. After comparative performance tests, restarted GMRES(15) taken 
Preprint 2007, Alfred Wegener Institute, Germany, M. Läuter, et al.

from PETSc preconditioned with global algebraic multigrid (BoomerAMG from hypre) is currently used, see section 6.4.

\section{Model validation}

PLASMA is validated by means of numerical experiments. The validation process is carried out in two steps. At first, a convergence study considering analytical solutions of the spherical shallow water equations is performed. Although these solutions are rather artificial, the distance to an analytical solution is the only possibility to evalutate the error of a numerical solution. After that, simulations of Rossby-Haurwitz waves and of planetary Rossby waves forced by orography have been carried out.

Two kinds of experiments have been performed; uniform grid experiments and adaptive grid experiments. In a uniform grid experiment the grid of the model remains temporally fixed. In an adaptive grid experiment the grid is adapted at every time step according to the physical error indicator described in section 4 .

The model simulations have been shown by performing adaptive grid experiments instead of uniform grid experiments, that up to $75 \%$ fewer grid points can be used still obtaining nearly the same accuracy. Certainly, the dimension of the problem size, that is proportional to the number of grid points, differs to the dimension of the computational costs. That is because adaptive grid experiments include more computations per grid point, for instance the computation of the error indicator and the grid adaptation. Further, a reliable comparison of computational costs, especially to methods already in use, is rather complicated, because the model code is optimized for adaptive grid experiments, but not for uniform ones.

\subsection{Analytical solutions}

In this section all numerical experiments have been carried out with an integration time of $T=5$ day. Various initial conditions have been given by different known analytical solutions. For the empirical convergence tests the relative $L^{2}$-errors $\eta(\Phi)$ of the geopotential have been considered. Therefore, $\eta(\Phi)$ has been computed for different grid resolutions. For the uniform grid experiments, the grid resolution $\Delta x$ has ranged from $261 \mathrm{~km}$ up to $1041 \mathrm{~km}$. For the adaptive grid experiments, the parameter $\Delta x$ indicates the finest grid resolution. The corresponding coarsest resolutions are denoted in table I. The time step $\Delta t$ is chosen such, that the CourantFriedrichs-Levy (CFL) number $u \frac{\Delta t}{\Delta x}$ equals $u \frac{900 \mathrm{~s}}{854 \mathrm{~km}}$ for all experiments in this section. This yields $u \frac{\Delta t}{\Delta x}<0.1$ with a maximum velocity of $80 \mathrm{~m} / \mathrm{s}$.

Numerical solutions converge to the analytical ones for the steady state case as well as for the unsteady case. In all experiments the experimental order of convergence is about 1.0, which seems to be appropriate due to the applied first order method in time, see the trajectory approximation in section 3.2. The adaptive grid experiments 
Preprint 2007, Alfred Wegener Institute, Germany, M. Läuter, et al.

\begin{tabular}{c|c|c|c|c|c}
$\Delta x$, finest resolution $[\mathrm{km}]$ & 261 & 428 & 522 & 854 & 1041 \\
\hline coarsest resolution $[\mathrm{km}]$ & 1041 & 1701 & 2058 & 3339 & 3938
\end{tabular}

Table I: Adaptive grid experiment, parameter $\Delta x$ with the range of grid resolution.

need between about a half and a quarter the number of grid points compared to the uniform grid experiment and show only slightly larger $L^{2}$-errors.

\subsubsection{Solid body rotations}

After writing down a general solid body rotation, a steady-state and an unsteady version are used to initialize two experiments. Therefore, let us choose arbitrarily (for the experimental set-up, see values below) a vector $\mathbf{c} \in \mathbb{R}^{3}$ with $|\mathbf{c}|=1$, a maximal velocity $u_{0}$ and a constant $d_{0}$. Then the solid body rotation

$$
\begin{aligned}
\mathbf{u}_{s b r}(\mathbf{x}, t) & =u_{0} \varphi_{t}(\mathbf{c}) \times \mathbf{k}, \\
\Phi_{s b r}(\mathbf{x}, t) & =\frac{-\left(u_{0} \varphi_{t}(\mathbf{c}) \cdot \mathbf{k}+\boldsymbol{\Omega} \cdot \mathbf{x}\right)^{2}}{2}+\frac{(\boldsymbol{\Omega} \cdot \mathbf{x})^{2}}{2}+d_{0}
\end{aligned}
$$

with the orography field

$$
\Phi_{B}(\mathbf{x})=\frac{(\boldsymbol{\Omega} \cdot \mathbf{x})^{2}}{2}
$$

is an analytical solution of the spherical shallow water equations, see [33, Example $3]$. Here, $\boldsymbol{\Omega}=(0,0, \Omega)^{\mathrm{T}}$ is the Earth's angular velocity vector and $\varphi_{t}$ with

$$
\varphi_{t}(\mathbf{c})=\left(\begin{array}{ccc}
\cos (\Omega t) & \sin (\Omega t) & 0 \\
-\sin (\Omega t) & \cos (\Omega t) & 0 \\
0 & 0 & 1
\end{array}\right) \mathbf{c}
$$

is a linear rotation map. With the choice of parameters

$$
\mathbf{c}=(0,0,1)^{\mathrm{T}}, \quad u_{0}=2 \pi a /(12 \text { day }), \quad d_{0}=29400 \mathrm{~m}^{2} / \mathrm{s}^{2}
$$

the functions $\mathbf{u}_{s b r}$ and $\Phi_{s b r}$ in Eq. (6) are a steady-state solution of the spherical shallow water equations, with orography given by Eq. (7) as well as with $\Phi_{B} \equiv$ 0 . This solution coincides with [49, test 2]. With the choice of parameters $\mathbf{c}=$ $\left(-\sin \frac{\pi}{4}, 0, \cos \frac{\pi}{4}\right)^{\mathrm{T}}, u_{0}=2 \pi a / 12 \mathrm{~m} /$ day, $d_{0}=133681 \mathrm{~m}^{2} / \mathrm{s}^{2}$ the functions $\mathbf{u}_{s b r}$ and $\Phi_{s b r}$ in Eq. (6) are an unsteady solution of the spherical shallow water equations, with orography given by Eq. (7).

The relative $L^{2}$-errors of the geopotential $\eta(\Phi)$ for the steady-state and unsteady solid body rotation are displayed in Fig. 3. Numerical solutions converge to the analytical solutions with experimental orders of convergence of about 1.01 and 0.76 , respectively. The uniform grid experiment uses 20482 points. The adaptive grid experiment uses 11331 (10300) grid points in the steady-state (unsteady) case at time $T=5$ day and for $\Delta x=261 \mathrm{~km}$. The corresponding relative $L^{2}$-errors are $2.4 \cdot 10^{-4}$ respectively $5.0 \cdot 10^{-5}$ greater than for the uniform case. Hence, the adaptive grid experiment needs only half the number of grid points, but leads to only a slightly greater $L^{2}$-error compared to the uniform grid experiment. 

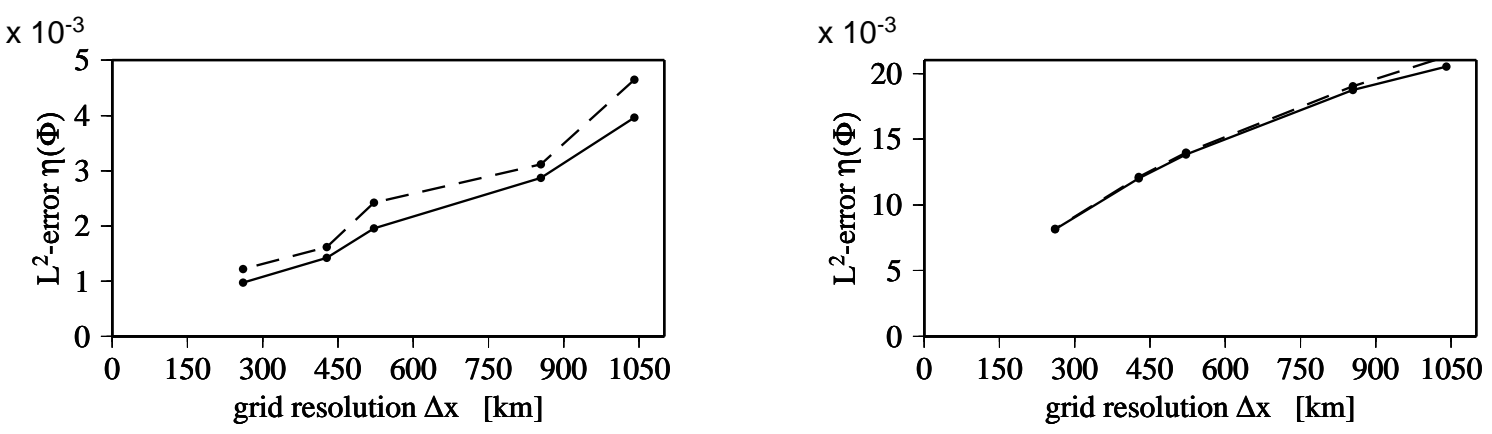

Figure 3: Solid body rotations, relative $L^{2}$-errors $\eta(\Phi)$ at time $T=5$ day; uniform grid experiments - solid line, adaptive grid experiments - dashed line; left: steadystate solution, right: unsteady solution.

\subsubsection{Jet streams}

In a similar way as in the preceding section, we introduce a general jet stream, whose steady-state and unsteady version is used to initialize two experiments. Therefore, let us choose arbitrarily a vector $\mathbf{c} \in \mathbb{R}^{3}$ with $|\mathbf{c}|=1$, maximal velocities $u_{0}, u_{1}$, latitudinal angles $\theta_{0}, \theta_{1}$ and and a constant $d_{0}$. The auxiliary axis a and the velocity profiles $u_{p, s b r}, u_{p, j e t}$ are defined by

$$
\begin{gathered}
\mathbf{a}=\frac{u_{0} \mathbf{c}+a \boldsymbol{\Omega}}{\left|u_{0} \mathbf{c}+a \boldsymbol{\Omega}\right|}, \quad u_{p, s b r}(x)=\left|u_{0} \mathbf{c}+a \boldsymbol{\Omega}\right| \sqrt{1-x^{2}}, \quad \forall x \in[-1,1], \\
u_{p, j e t}(\sin \theta)= \begin{cases}u_{1} \exp \left(\frac{1}{\left(\theta-\theta_{0}\right)\left(\theta-\theta_{1}\right)}+\frac{4}{\left(\theta_{0}-\theta_{1}\right)^{2}}\right) & \text { for } \theta_{0} \leq \theta \leq \theta_{1}, \\
0 & \text { else }\end{cases}
\end{gathered}
$$

where $a$ denotes the Earth radius given in section 2. The jet stream

$$
\begin{aligned}
\mathbf{u}(\mathbf{x}, t) & =\mathbf{u}_{s b r}(\mathbf{x}, t)+u_{p, j e t}\left(\varphi_{t}(\mathbf{a}) \cdot \mathbf{k}\right) \frac{\varphi_{t}(\mathbf{a}) \times \mathbf{k}}{\left|\varphi_{t}(\mathbf{a}) \times \mathbf{k}\right|}, \\
\Phi(\mathbf{x}, t) & =\Phi_{s b r}(\mathbf{x}, t)-\int_{0}^{\arcsin \left(\varphi_{t}(\mathbf{a}) \cdot \mathbf{k}\right)} \tan \phi\left(2 u_{p, s b r} u_{p, j e t}+u_{p, j e t}^{2}\right) \circ \sin \theta d \theta+d_{0}
\end{aligned}
$$

with orography given by Eq. (7) is an analytical solution of the spherical shallow water equations, see [33, Example 4]. With the choice of parameters

$$
\begin{aligned}
& \mathbf{c}=(0,0,1)^{\mathrm{T}}, \quad u_{0}=0, \quad u_{1}=2 \pi a /(12 \text { day }), \\
& \theta_{0}=\frac{\pi}{18}, \quad \theta_{1}=\frac{5 \pi}{18}, \quad d_{0}=98100 \mathrm{~m}^{2} / \mathrm{s}^{2}
\end{aligned}
$$

the functions $\mathbf{u}$ and $\Phi$ in Eq. (9) are a steady-state solution of the spherical shallow water equations, with orography given by Eq. (7) as well as with $\Phi_{B} \equiv 0$. This solution is similar to the undisturbed initial fields in [21]. With the choice of parameters $\mathbf{c}=\left(-\sin \frac{\pi}{4}, 0, \cos \frac{\pi}{4}\right)^{\mathrm{T}}, u_{0}=20 \mathrm{~m} / \mathrm{s}, u_{1}=2 \pi a /(12$ day $), \theta_{0}=\frac{\pi}{18}, \theta_{1}=\frac{5 \pi}{18}$ 

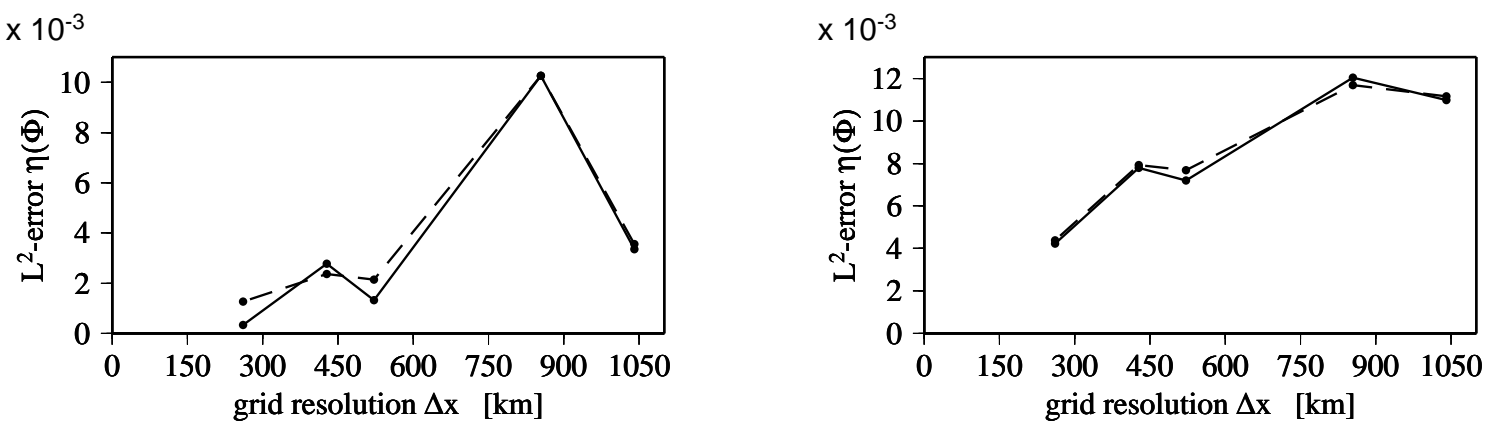

Figure 4: Jet streams, relative $L^{2}$-errors $\eta(\Phi)$ at time $T=5$ day; uniform grid experiments - solid line, adaptive grid experiments - dashed line; left: steady-state solution, right: unsteady solution.

and $d_{0}=129629 \mathrm{~m}^{2} / \mathrm{s}^{2}$ the functions $\mathbf{u}$ and $\Phi$ in Eq. (9) are an unsteady solution of the spherical shallow water equations, with orography given by Eq. (7).

The relative $L^{2}$-errors of the geopotential $\eta(\Phi)$ for the steady-state and the unsteady jet stream are displayed in Fig. 4. The significant oscillations of $\eta(\Phi)$ are a model artefact which can be attributed to the anisotropic structure of the triangular grid, see [33] for a detailed explanation. Numerical solutions converge to the analytical solutions with the experimental orders of convergence of about 1.9 and 0.77 , respectively. The uniform grid experiment uses 20482 points. The adaptive grid experiment uses 5500 (5923) grid points in the steady-state (unsteady) case at time $T=5$ day and for $\Delta x=261 \mathrm{~km}$. The corresponding relative $L^{2}$-errors are $9.4 \cdot 10^{-4}$ respectively $1.7 \cdot 10^{-4}$ greater than for the uniform case. Hence, similar as in section 6.1.1 the adaptive grid experiment needs only a quarter the number of grid points, but leads to an only slightly greater $L^{2}$-error compared to the uniform grid experiment.

\subsection{Rossby-Haurwitz waves}

Rossby-Haurwitz waves were first used for the validation of a shallow water model by Phillips [38]. These are solutions of the linearized non-divergent barotropic vorticity equation and move from west to east without change of their shape. Here, a RossbyHaurwitz wave with wave number $R=4$ has been studied. Beside the contour plots, conservation properties of the model have been evaluated. A uniform grid experiment has been performed with the grid resolution $\Delta x=131 \mathrm{~km}$ and the time step $\Delta t=137 \mathrm{~s}$, which corresponds to the CFL number $u \frac{900 \mathrm{~s}}{854 \mathrm{~km}}$ in section 6.1 .

Rossby-Haurwitz waves, see [49, Test case 6] for a complete description, are given by their zonal and meridional wind fields

$$
\begin{aligned}
& u(\lambda, \theta)=a \omega \cos \theta+a \omega \cos ^{R-1} \theta\left(R \sin ^{2} \theta-\cos ^{2} \theta\right) \cos R \lambda, \\
& v(\lambda, \theta)=-a \omega R \cos ^{R-1} \theta \sin \theta \sin R \lambda,
\end{aligned}
$$

with the constant $\omega=7.848 \cdot 10^{-6} 1 / \mathrm{s}$ and the longitudinal and latitudinal angles $\lambda$ and $\theta$, respectively. In vector formulation, this can be reproduced by 

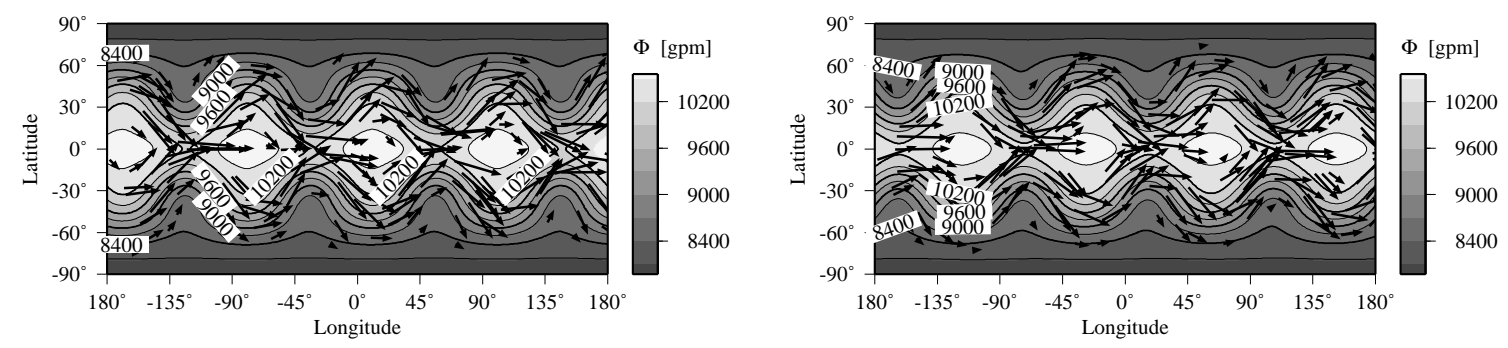

Figure 5: Rossby-Haurwitz wave, contour plots for geopotential $\Phi$ after 1 and 14 days.
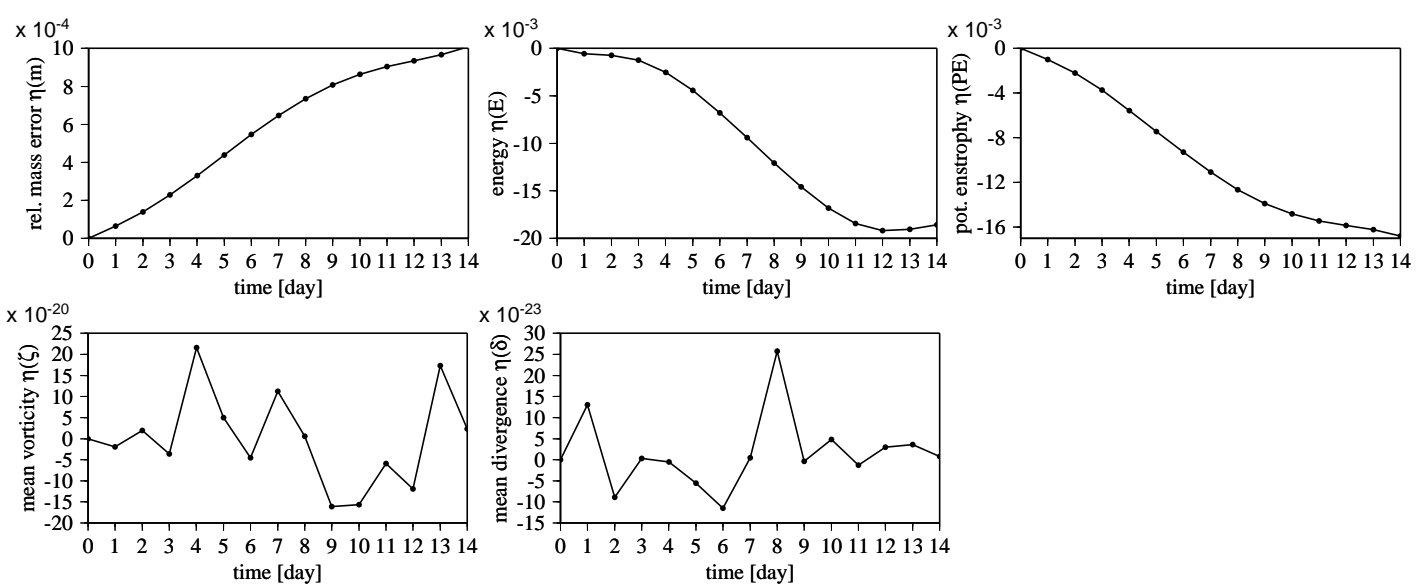

Figure 6: Rossby-Haurwitz wave, rel. errors of mass, total energy and potential enstrophy and mean values of vorticity and divergence.

$\mathbf{u}(\mathbf{x}, 0)=u(\lambda, \theta) \mathbf{i}+v(\lambda, \theta) \mathbf{j}$ with the unit vectors $\mathbf{i}$ and $\mathbf{j}$ into eastward and northward direction, respectively.

Contour plots for the geopotential $\Phi$ are depicted in Fig. 5, for simulation times of 1 and 14 days, respectively. The large-scale wave structure is temporally conserved, although the meridional wave amplitude is reduced due to numerical diffusion of the model. The values of mass $m$, total energy $E$, potential enstrophy $P E$, vorticity $\zeta$ and divergence $\delta$ are given in Fig. 6, see e.g. [49] for the definitions of the variables. While the relative errors of the global integrals are plotted for $m, E$ and $P E$, the mean values are displayed for vorticity and divergence. As a consequence of the semi-Lagrangian approach neither $m$ nor $E$ nor $P E$ are conserved in the experiment. After 14 days, these grid size dependent errors are close to $0.1 \%, 2 \%$ and $1.5 \%$, for $m, E$ and $P E$ respectively. $\zeta$ and $\delta$ are prognostic model variables and occur as right hand sides of the Poisson equations in (3). Hence, the mean values are independent on the grid size and close to machine precision.

\subsection{Zonal flow over a mountain}

The orographic forcing of planetary Rossby waves has been studied by means of 15 day long experiments, initialized with two different flow fields, a solid body ro- 
Preprint 2007, Alfred Wegener Institute, Germany, M. Läuter, et al.
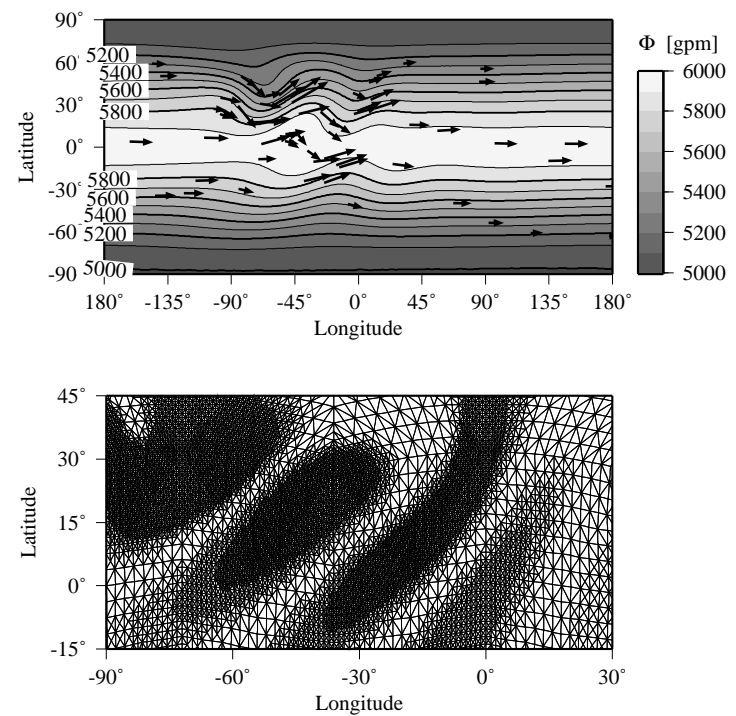

Figure 7: Solid body rotation with mountain, adaptive grid experiment; contour plot of geopotential $\Phi$ and computational grid; time $T=5$ day.

tation and a jet stream. These zonal fields are disturbed by an isolated mountain generating meridional disturbances and therewith planetary waves. The orography of the mountain is given by $\Phi_{B}(\mathbf{x})=\Phi_{\max }(1-r(\mathbf{x}))$ with the auxiliary function $r(\mathbf{x})=\min \left(1, \frac{9}{\pi} \sqrt{\left(\lambda(\mathbf{x})+\frac{\pi}{2}\right)^{2}+\left(\theta(\mathbf{x})-\frac{\pi}{6}\right)^{2}}\right)$ and the mountain height $\Phi_{\text {max }}=2000 \mathrm{gpm}$, where $1 \mathrm{gpm}=9.81 \mathrm{~m}^{2} / \mathrm{s}^{2}$.

For both initial fields a uniform grid experiment and an adaptive grid experiment have been performed. The time step is $\Delta t=900 \mathrm{~s}$, whereas the grid resolution amounts to $131 \mathrm{~km}$ for the uniform grid experiments and ranges from $131 \mathrm{~km}$ up to $522 \mathrm{~km}$ for the adaptive grid experiments.

\subsubsection{Solid body rotation}

The first experiment is in accordance with test case 5 in [49]. The simulation has been initialized with the steady-state solid body rotation of section 6.1.1, but with constants $u_{0}=20 \mathrm{~m} / \mathrm{s}$ and $d_{0}=58468 \mathrm{~m}^{2} / \mathrm{s}^{2}$. The contour plots for geopotential $\Phi$ are depicted in the Figs. 7 and 8 for the adaptive grid experiment after 5 and 15 days. Orographic forcing by the isolated mountain is visible. The disturbance propagates into south-easterly direction, thus the planetary waves develop into the southern hemisphere, too. The bottom subplots in the Figs. 7 and 8 show sections of the corresponding grids. The time dependent regions of higher resolution follow the wave structure in the geopotential.

After 15 days, the adaptive grid experiment utilizes 21528 points, whereas the uniform grid experiment utilizes 81922 points. The geopotential difference fields between the adaptive grid experiment and the uniform grid experiment are depicted in Fig. 9. While the differences after 5 days are below 4 gpm they have been grown 
Preprint 2007, Alfred Wegener Institute, Germany, M. Läuter, et al.
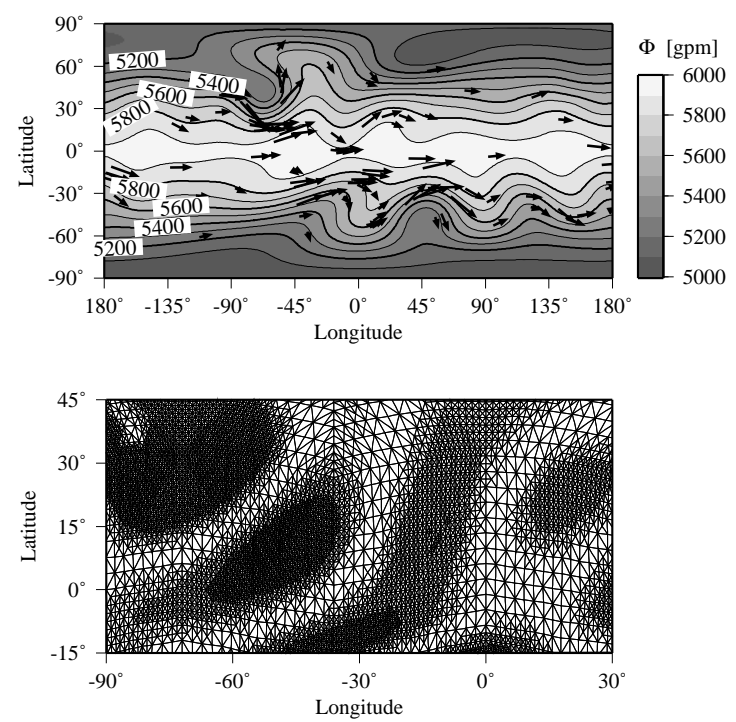

Figure 8: Solid body rotation with mountain, adaptive grid experiment; contour plot of geopotential $\Phi$ and computational grid; time $T=15$ day.
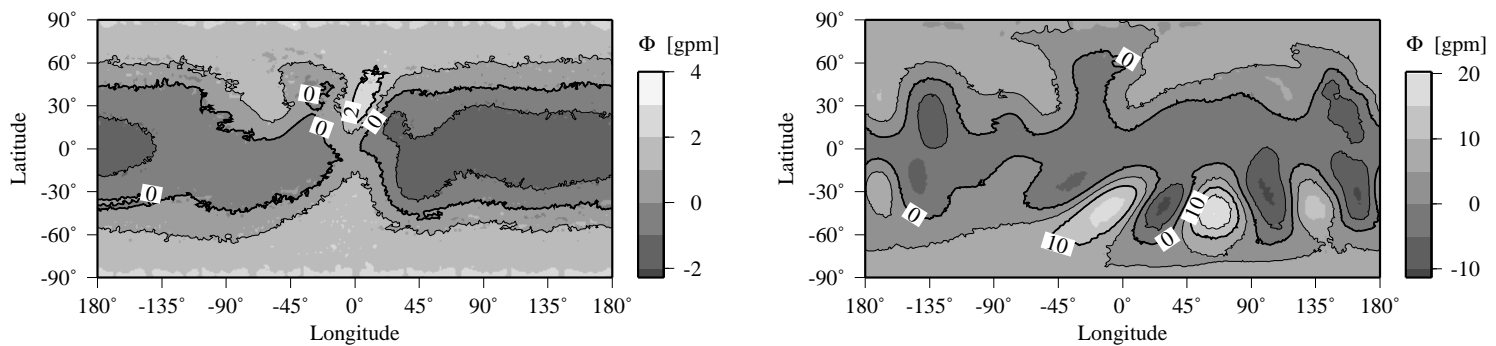

Figure 9: Solid body rotation with mountain, "adaptive grid experiment" minus "uniform grid experiment"; contour plots of geopotential $\Phi$; left: time $T=5$ day, right: time $T=15$ day.

up to $20 \mathrm{gpm}$ after 15 days in the eddies at $45^{\circ} \mathrm{S}$. Because of a layer depth of more than $5000 \mathrm{gpm}$, a quarter the number of grid points in the adaptive grid experiment is sufficient to obtain a geopotential difference smaller than $0.5 \%$ compared to the uniform grid experiment.

\subsubsection{Jet stream}

The second experiment has been initialized with the steady-state jet stream introduced in section 6.1.2, see [32]. The contour plots for the geopotential $\Phi$ are depicted in the Figs. 10 and 11 for the adaptive grid experiment after 5 and 15 days. Like in section 6.3.1, the orographic forcing by the isolated mountain is visible. Now, the disturbance propagates mainly into easterly direction due to the jet structure. Thus, the planetary waves develop along the jet stream in the northern hemisphere only. The bottom subplots in the Figs. 10 and 11 show sections of the correspond- 
Preprint 2007, Alfred Wegener Institute, Germany, M. Läuter, et al.
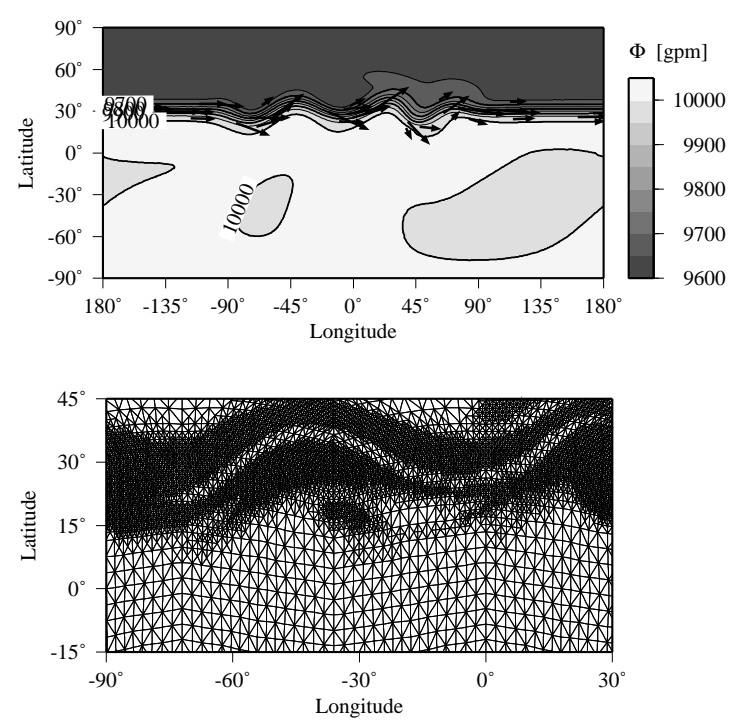

Figure 10: Jet stream with mountain, adaptive grid experiment; contour plot of geopotential $\Phi$ and computational grid; time $T=5$ day.

ing grids. Again, the time dependent regions of higher resolution follow the wave structure in the geopotential. Beside the planetary wave structure, refined regions outside the original jet regions are visible. This indicates a finer resolution due to turbulent flow structures and eddies.

After 15 days, the adaptive grid experiment utilizes 17796 points, whereas the uniform grid experiment utilizes 81922 points. The geopotential difference fields between the adaptive grid experiment and the uniform grid experiment are depicted in Fig. 12. After 5 days the maximum geopotential differences are smaller than $16 \mathrm{gpm}$, but they grow up to about $60 \mathrm{gpm}$ after 15 days in the region of large eddies at $30^{\circ} \mathrm{N}$. Due to a layer depth of more than $9500 \mathrm{gpm}$, this corresponds to a relative error of approximately $0.6 \%$. Although the highest grid resolution is near the eddies, the maximum error is located there. This could be an indication for the fact, that the physical error indicator detects a refinement area as too small to reproduce the turbulent flow structures absolutely correct. Nevertheless, a quarter the number of grid points in the adaptive grid experiment is sufficient to simulate planetary waves, that differ only $0.6 \%$ to the results in the uniform grid experiment.

\subsection{Performance data for linear solver}

In this section we present performance data of standard parallel solvers from PETSc 2.2.1/hypre 1.8.2b and MUMPS 4.3.2 assessed via FoSSI as introduced in section 5. They are applied to solve the Poisson equations in (3) within the adaptive grid experiment in section 6.3.2, that is a jet stream overflowing an isolated mountain. In this section the grid resolution ranges from $33 \mathrm{~km}$ up to $1041 \mathrm{~km}$ yielding 
Preprint 2007, Alfred Wegener Institute, Germany, M. Läuter, et al.
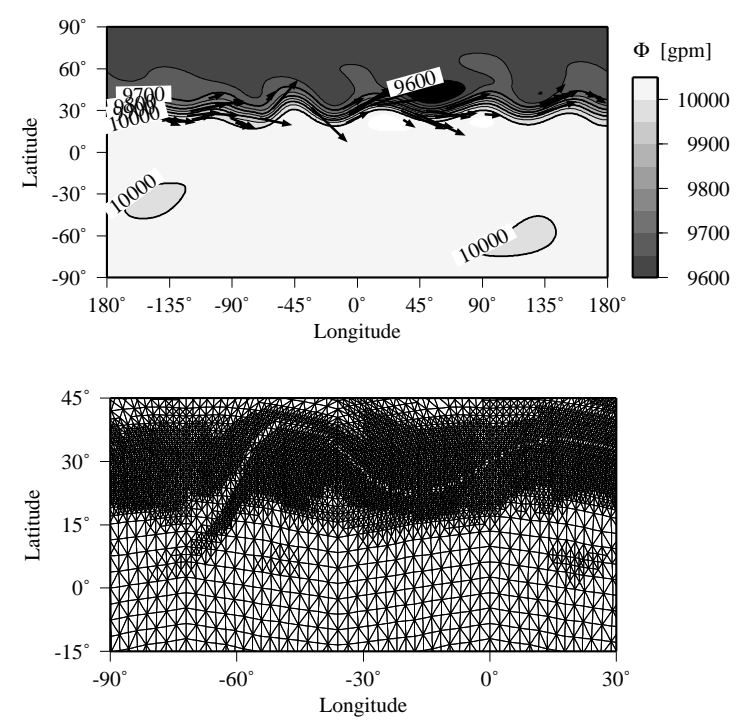

Figure 11: Jet stream with mountain, adaptive grid experiment; contour plot of geopotential $\Phi$ and computational grid; time $T=15$ day.
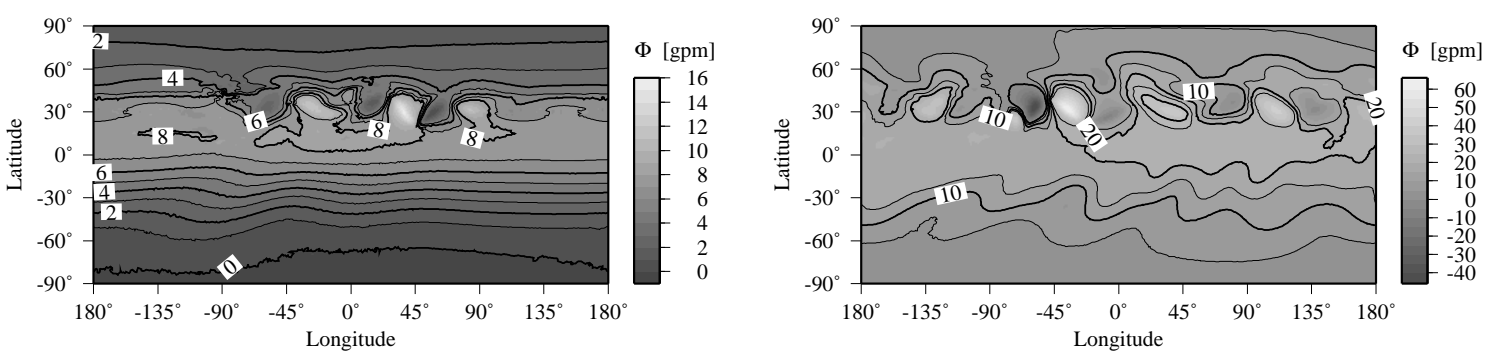

Figure 12: Jet stream with mountain, "adaptive grid experiment" minus "uniform grid experiment"; contour plots of geopotential $\Phi$; left: time $T=5$ day, right: time $T=15$ day. 
Preprint 2007, Alfred Wegener Institute, Germany, M. Läuter, et al.

a matrix with 572158 rows. This ratio of maximum to minimum grid resolution clearly exceeds the ratio for the adaptive grid experiments in the sections 6.1 and 6.3. The performance data show that the solver accomplishes even this demanding situation.

Performance data of solving the linear system using GMRES from PETSc (restarted every 15 iterations) in combination with three different preconditioners are presented for two to eight CPUs in table II. The first column describes the preconditioner. AMGglob stands for global algebraic multigrid (AMG, from hypre) preconditioning, A2AMGloc stands for restricted additive Schwarz method with overlap 2 in combination with local AMG iterations and A2ILU3 stands for restricted additive Schwarz method in combination with a level 3 incomplete factorization from PETSc. As the matrix is symmetric, a combination of a symmetric incomplete factorization and a CG method was also tested. The results are omitted because this combination yielded only minor performance. The second and the third columns contain the number of CPUs and the iteration counts (\#Its) for the solution task, respectively. $t_{P C}$ and $t_{S o l}$ give the times for the preconditioner setup and the solver in seconds. The parallel efficiency $\mathrm{P}_{\text {eff }}$ of the solver, indicating the relation of observed speedup to optimal (linear) speedup, is given in the last column (based on the sum of the time needed for the preconditioner setup and the solver $\left.t_{P C}+t_{S o l}\right)$. All computations were performed on one node of an IBM Regatta p655 with 8 Power4+ processors at $1.7 \mathrm{GHz}$.

\begin{tabular}{l|r|r|r|r|r} 
PC & CPUs & \#Its & $t_{P C}$ & $t_{\text {Sol }}$ & $\mathrm{P}_{\text {eff }}$ \\
\hline \hline AMGglob & 2 & 14 & $5.05 \mathrm{~s}$ & $6.94 \mathrm{~s}$ & - \\
AMGglob & 4 & 13 & $2.59 \mathrm{~s}$ & $3.43 \mathrm{~s}$ & $99.5 \%$ \\
AMGglob & 8 & 12 & $1.36 \mathrm{~s}$ & $1.94 \mathrm{~s}$ & $91.8 \%$ \\
\hline A2AMGloc & 2 & 112 & $4.86 \mathrm{~s}$ & $50.11 \mathrm{~s}$ & - \\
A2AMGloc & 4 & 172 & $2.48 \mathrm{~s}$ & $39.94 \mathrm{~s}$ & $65.0 \%$ \\
A2AMGloc & 8 & 234 & $1.33 \mathrm{~s}$ & $33.05 \mathrm{~s}$ & $40.0 \%$ \\
\hline A2ILU3 & 2 & 627 & $8.95 \mathrm{~s}$ & $246.79 \mathrm{~s}$ & - \\
A2ILU3 & 4 & 628 & $4.55 \mathrm{~s}$ & $129.76 \mathrm{~s}$ & $94.5 \%$ \\
A2ILU3 & 8 & 581 & $2.40 \mathrm{~s}$ & $71.34 \mathrm{~s}$ & $86.0 \%$
\end{tabular}

Table II: Comparison of different parallel preconditioners from PETSc/hypre on IBM p655

The convergence criterion is the relative reduction of the residual norm of $10^{-11}$. The AMGglob combination has the smallest number of iterations with only 13 compared to 172 and 628 for A2AMGloc and A2ILU3, respectively. This is represented in the timings, too. AMGglob is much faster than any of the other combinations. Simultaneously, the parallel efficiency shows very good results.

After choosing GMRES(15) with global AMG preconditioning (GMRES/AMGglob) as an appropriate iterative solver for the Poisson equation, this combination is compared to the direct solver MUMPS, see table III. To allow a more detailed parallel 
Preprint 2007, Alfred Wegener Institute, Germany, M. Läuter, et al.

analysis these tests have been carried out on two 32-processor-nodes of an IBM p690 with $1.3 \mathrm{GHz}$ Power4 processors with a high performance switch interconnect. The columns CPUs, \#Its, $t_{P C}$ and $t_{S o l}$ in table III have the same meaning as in table II. As the time for the setup of the matrix structure in MUMPS is significantly larger than in GMRES/AMGglob, the total time of the solver call $t_{\text {Call }}$ is considered beside the factorization time $t_{P C}$ and the solution time $t_{S o l}$. This is especially important in an adaptive model where the mesh and hence the nonzero pattern of the matrix may change in every time step. The parallel efficiencies $\mathrm{P}_{\text {eff }}$ are calculated based on the time $t_{\text {Call }}$.

\begin{tabular}{l|r|r|r|r|r|r} 
Solver & CPUs & \#Its & $t_{P C}$ & $t_{\text {Sol }}$ & $t_{\text {Call }}$ & $\mathrm{P}_{\text {eff }}$ \\
\hline \hline GMRES/AMGglob & 2 & 36 & $6.50 \mathrm{~s}$ & $22.12 \mathrm{~s}$ & $29.37 \mathrm{~s}$ & - \\
GMRES/AMGglob & 8 & 24 & $1.66 \mathrm{~s}$ & $3.44 \mathrm{~s}$ & $5.32 \mathrm{~s}$ & $138 \%$ \\
GMRES/AMGglob & 16 & 23 & $0.97 \mathrm{~s}$ & $2.07 \mathrm{~s}$ & $3.21 \mathrm{~s}$ & $115 \%$ \\
GMRES/AMGglob & 32 & 18 & $0.74 \mathrm{~s}$ & $1.67 \mathrm{~s}$ & $2.59 \mathrm{~s}$ & $71 \%$ \\
GMRES/AMGglob & 64 & 18 & $0.58 \mathrm{~s}$ & $1.29 \mathrm{~s}$ & $2.15 \mathrm{~s}$ & $43 \%$ \\
\hline MUMPS & 2 & & $6.19 \mathrm{~s}$ & $3.94 \mathrm{~s}$ & $13.22 \mathrm{~s}$ & - \\
MUMPS & 8 & & $3.21 \mathrm{~s}$ & $2.32 \mathrm{~s}$ & $8.74 \mathrm{~s}$ & $38 \%$ \\
MUMPS & 16 & & $2.59 \mathrm{~s}$ & $3.21 \mathrm{~s}$ & $9.08 \mathrm{~s}$ & $18 \%$ \\
MUMPS & 32 & & $2.55 \mathrm{~s}$ & $2.86 \mathrm{~s}$ & $8.94 \mathrm{~s}$ & $9 \%$ \\
MUMPS & 64 & & $2.52 \mathrm{~s}$ & $2.90 \mathrm{~s}$ & $9.32 \mathrm{~s}$ & $4 \%$
\end{tabular}

Table III: Comparison on IBM p690: GMRES(15) preconditioned with global AMG vs. direct solver MUMPS

The reduction of the residual norm of $10^{-15}$ was stipulated, as this is approximately the accuracy the direct solver achieves. It is seen from the parallel efficiencies $\mathrm{P}_{\text {eff }}$ that the scalability of MUMPS is rather limited while GMRES/AMGglob has the potential for efficient application on massively parallel systems ( $\geq 32 \mathrm{CPUs}$ ). The degradation of the performance from 32 to 64 CPUs on the IBM p690 can be explained by its architecture as the calculation with 64 CPUs is the only one that is performed between two shared memory nodes of the machine. Further tests on a Cray XD1 with a non hierarchical CPU interconnect even show a parallel efficiency of $68 \%$ for GMRES/AMGglob on 48 CPUs.

These performance experiments demonstrate that the combination of restarted GMRES(15) from PETSc and global algebraic multigrid (BoomerAMG from hypre) is the best solver for the Poisson equations in (3). This combination outperforms other combinations of the iterative solver as well as even the direct solver MUMPS.

\section{Summary}

PLASMA is a parallel adaptive model of the atmosphere. For the discretization of the underlying spherical shallow water equations a Lagrange-Galerkin method, a 
Preprint 2007, Alfred Wegener Institute, Germany, M. Läuter, et al.

combination of the finite element method and the semi-Lagrangian method, has been employed. The unstructured triangular grid is generated with the grid generator amatos and the large linear systems are solved with the parallel solver interface FoSSI.

Both, uniform grid experiments as well as adaptive grid experiments can be performed with PLASMA. In the adaptive case the computational grid is adapted at every time step according to a physical error indicator. The comparison of uniform and adaptive grid experiments documents, that the adaptive model leads to a significant reduction of the number of grid points while the numerical error increases only slightly. This has been shown within convergence studies for steady-state and unsteady analytical solutions as well as for zonal flow over an isolated mountain. In other words, if one would allow the same number of grid points for a uniform respectively an adaptive grid experiment, the adaptive one would yield more accurate results for local features of interest, compared to the uniform grid experiment.

By means of a sample of quasi standard experiments the successful numerical approximation of the spherical shallow water equations has been shown. Convergence studies show the first order approximation for steady-state and unsteady analytical solutions. Furthermore, PLASMA shows satisfactory results for the simulation of Rossby-Haurwitz waves and zonal flows over an isolated mountain.

For the realization of adaptive simulations from seasonal up to annual time scales within a simplified dynamical model of the atmosphere, PLASMA still needs improvement. Longer model integrations require a discrete conservation of the physical variables mass, energy and potential enstrophy. Although, the presented simulations already show very satisfactory results for adaptive simulations, the physical error indicator should be further improved, especially inside turbulent flow structures. Finally, the application of the adaptive grid in PLASMA can be assigned to a baroclinic multi-layer model, assuming the same horizontal grid structure throughout all vertical layers.

\section{Acknowledgments}

The authors thank Florian Klaschka for (parallel) code optimization and code restructuring. Support by the Federal Ministry of Education and Research of Germany in the framework of DEKLIM (German climate research program, 01 LD 0037) is gratefully acknowledged. The authors thank the two anonymous reviewers for useful hints which improved the manuscript. 
Preprint 2007, Alfred Wegener Institute, Germany, M. Läuter, et al.

\section{References}

[1] P. R. Amestoy, I. S. Duff, and J.-Y. L'Excellent. Multifrontal parallel distributed symmetric and unsymmetric solvers. Comput. Methods in Appl. Mech. Eng., 184:501-520, 2000.

[2] D. P. Bacon, N. N. Ahmad, Z. Boybeyi, T. J. Dunn, M. S. Hall, P. C. S. Lee, R. A. Sarma, and M. D. Turner. A dynamically adapting weather and dispersion model: The operational multiscale environment model with grid adaptivity (OMEGA). Mon. Wea. Rev., 128:2044-2076, 2000.

[3] S. Balay, K. Buschelmann, V. Eijkhout, W. Gropp, D. Kaushik, M. Knepley, L. C. McInnes, B. Smith, and H. Zhang. PETSc Users Manual. Argonne National Labratory, August 2004. Version 2.2.1.

[4] E. Bänsch. Local mesh refinement in 2 and 3 dimensions. Impact of Comput. in Sci. and Eng., 3:181-191, 1991.

[5] S. R. M. Barros and C. I. Garcia. A global semi-implicit semi-Lagrangian shallow-water model on locally refined grids. Mon. Wea. Rev., 132:53-65, 2004.

[6] J. Behrens. Adaptive mesh generator for atmospheric and oceanic simulations amatos. Technical Report TUM-M0409, Technische Universität München, Zentrum Mathematik, Boltzmannstr. 3, 85747 Garching, 2004. URL: http://wwwlit.ma.tum.de/veroeff/html/040.65008.html.

[7] J. Behrens, N. Rakowsky, W. Hiller, D. Handorf, M. Läuter, J. Päpke, and K. Dethloff. amatos: Parallel adaptive mesh generator for atmospheric and oceanic simulation. Ocean Modelling, 10:171-183, 2005.

[8] J. Behrens and J. Zimmermann. Parallelizing an unstructured grid generator with a space-filling curve approach. In A. Bode, T. Ludwig, W. Karl, and R. Wismüller, editors, Euro-Par 2000 Parallel Processing - 6th International Euro-Par Conference Munich, Germany, August/Sptember 2000 Proceedings, volume 1900 of Lecture Notes in Computer Science, pages 815-823, Berlin, 2000. Springer Verlag.

[9] L. Bonaventura and T. R. Ringler. Analysis of Discrete Shallow-Water Models on Geodesic Delauny Grids with C-Type Staggering. Mon. Wea. Rev., 133:2351-2373, 2004.

[10] J. Côté. A Lagrange multiplier approach for the metric terms of semiLagrangian models on the sphere. Q. J. R. Meteorol. Soc., 114:1347-1352, 1988.

[11] P. Courtier and J. F. Geleyn. A global numerical weather prediction model with variable resolution: Application to the shallow water equations. Q. J.R. Meteorol. Soc., 114:1321-1346, 1988. 
Preprint 2007, Alfred Wegener Institute, Germany, M. Läuter, et al.

[12] H. C. Davies. A lateral boundary formulation for multi-level prediction models. Q. J. R. Meteorol. Soc., 102:405-418, 1976.

[13] M. Deque and J. P. Piodelievre. High resolution climate stimulation over europe. Clim. Dyn., 11:321-339, 1995.

[14] G. S. Dietachmayer and K. K. Droegemeier. Applications of continuous dynamic grid adaptation techniques to meteorological modeling. Part I: Basic formulation and accuracy. Mon. Wea. Rev., 120:1675-1706, 1992.

[15] R. D. Falgout and U. M. Yang. hypre: a Library of High Performance Preconditioners. In P. Sloot, C. Tan., J. Dongarra, and A. Hoekstra, editors, Computationla Science -ICCS 2002 Part III, volume 2331 of Notes in Computer Science, pages 632-641, 2002. Also available as Lawrence Livermore National Laboratory technical report UCRL-JC-146175 under http://www.llnl.gov/CASC/linear_solvers/pubs/hypre.ps.

[16] A. Fournier, M. A. Taylor, and J. J. Tribbia. The spectral element atmospheric model: High-resolution parallel computation and response to regional forcing. Mon. Wea. Rev., 132:726-748, 2004.

[17] M. S. Fox-Rabinovitz. Stretched-Grid Model Intercomparison Project, 2006. http://essic.umd.edu/ foxrab/sgmip.html.

[18] M. S. Fox-Rabinovitz, G. L. Stenchikov, M. J. Suarez, and L. L. Takacs. A finite-difference GCM dynamical core with a variable-resolution stretched grid. Mon. Wea. Rev., 125:2943-2968, 1997.

[19] M. S. Fox-Rabinovitz, L. Takacs, and R. Govindaraju. A variable resolution stretched grid general circulation model and data assimilation system with multiple areas of interest: Studying anomalous regional climate events of 1998. J. Geophys. Res., 107:4768, doi:10.1029/2002JD002177, 2002.

[20] S. Frickenhaus, W. Hiller, and M. Best. Fossi : The family of simplified solver interfaces for the rapid development of parallel numerical atmosphere and ocean models. Ocean Modelling, 10(1-2):185-191, 2005.

[21] J. Galewsky, R. Scott, and L. M. Polvani. An initial-value problem for testing numerical models of the global shallow water equations. 56:429-440, 2004.

[22] F. Giorgi. Simulation of regional climate using a limited area model nested in a general circulation model. J. Climate., 3:941-963, 1990.

[23] F. X. Giraldo and T. Warburton. A nodal triangle-based spectral element method for the spherical shallow water equations on unstructured grids. $J$. Comput. Phys., 207:129-150, 2005.

[24] G. J. Haltiner and R. Williams. Numerical Prediction and Dynamic Meteorology. Wiley, New York, 1980. 
Preprint 2007, Alfred Wegener Institute, Germany, M. Läuter, et al.

[25] V. Hardiker. A global numerical weather prediction model with variable resolution. Mon. Wea. Rev., 125:59-73, 1997.

[26] T. Heinze and A. Hense. The shallow water equations on the sphere and their Lagrange-Galerkin-solution. Meteorol. Atmos. Phys., 81:129-137, 2002.

[27] J. P. Iselin, J. M. Prusa, and W. J. Gutowski. Dynamic grid adaptation using the MPDATA scheme. Mon. Wea. Rev., 130:1026-1039, 2002.

[28] C. Jablonowski. Adaptive Grids in Weather and Climate Modeling. PhD thesis, University of Michigan, Ann Arbor, MI, USA, 2004.

[29] C. Jablonowski, M. Herzog, J. E. Penner, R. C. Oehmke, Q. F. Stout, and B. van Leer. Adaptive grids for weather and climate models. In ECMWF Seminar Proceedings on Recent Developments in Numerical Methods for Atmospheric and Ocean Modelling, pages 233-250, Reading, UK, 2004. ECMWF.

[30] G. Karypis and V. Kumar. Parallel Threshold-based ILU Factorization. In Proceedings of 9th Supercomputing Conference, 1997. see http://wwwusers.cs.umn.edu $/{ }^{\sim}$ karypis/publications/Papers/PDF/pilut.pdf.

[31] H.-J. Lange. Die Physik des Wetters und des Klimas. Dietrich Reimer Verlag, Berlin, 2002.

[32] M. Läuter. Großräumige Zirkulationsstrukturen in einem nichtlinearen adaptiven Atmosphärenmodell. PhD thesis, Universität Potsdam, Am Neuen Palais 10, 14469 Potsdam, Germany, 2004.

[33] M. Läuter, D. Handorf, and K. Dethloff. Unsteady analytical solutions of the spherical shallow water equations. J. Comput. Phys., 210:535-553, 2005.

[34] P. Lorenz and D. Jacob. Influence of regional scale information on the global circulation: A two-way nesting climate simulation. Geophys. Res. Lett., 32:L18706, doi:10.1029/2005GL023351, 2005.

[35] J. L. McGregor. Semi-Lagrangian advection on conformal-cubic grids. Mon. Wea. Rev., 124:1311-1322, 1996.

[36] K. W. Morton, A. Priestley, and E. Süli. Stability of the Lagrange-Galerkin Method with non-exact Integration. Math. Mod. Num. Anal., 22:625-653, 1988.

[37] J. Pedlosky. Geophysical Fluid Dynamics. Springer, New York, 1987.

[38] N. A. Phillips. Numerical integration of the primitive equations on the hemisphere. Mon. Wea. Rev., 87:333-345, 1959.

[39] J. M. Prusa and P. K. Smolarkiewicz. An all-scale anelastic model for geophysical flows: Dynamic grid deformation. J. Comput. Phys., 190:601-622, 2003. 
Preprint 2007, Alfred Wegener Institute, Germany, M. Läuter, et al.

[40] M. C. Rivara. Algorithms for refining triangular grids suitable for adaptive and multigrid techniques. International Journal for Numerical Methods in Engineering, 20:745-756, 1984.

[41] J. W. Ruge, S. F. McCormick, and S. Y. K. Yee. Multilevel adaptive methods for semi-implicit solution of shallow-water equations on the sphere. Mon. Wea. Rev., 123:2197-2205, 1995.

[42] F. Schmidt. Variable fine mesh in a spectral global model. Beitr. Phys. Atmos., 50:211-217, 1977.

[43] W. C. Skamarock and J. B. Klemp. Adaptive grid refinements for twodimensional and three-dimensional nonhydrostatic atmospheric flow. Mon. Wea. Rev., 121:788-804, 1993.

[44] W. C. Skamarock, J. Oliger, and R. L. Street. Adaptive grid refinements for numerical weather prediction. J. Comput. Phys., 80:27-60, 1989.

[45] A. Staniforth and J. Côté. Semi-Lagrangian Integration Schemes for Atmospheric Models-A Review. Mon. Wea. Rev., 119:2206-2223, 1991.

[46] A. N. Staniforth and H. L. Mitchell. A variable-resolution finite-element technique for regional forecasting with the primitive equations. Mon. Wea. Rev., 106:439-447, 1978.

[47] E. Süli. Convergence and Nonlinear Stability of the Lagrange-Galerkin Method for the Navier-Stokes Equations. Numer. Math., 53:459-483, 1988.

[48] R. S. Tuminaro, M. Heroux, S. A. Hutchinson, and J. N. Shadid. Official Aztec User's Guide: Version 2.1, 1999. http://www.cs.sandia.gov/CRF/aztec1.html.

[49] D. L. Williamson, J. B. Drake, J. J. Hack, R. Jakob, and P. N. Swarztrauber. A standard test set for numerical approximations to the shallow water equations in spherical geometry. J. Comput. Phys., 102:211-224, 1992.

[50] D.-L. Zhang, H.-R. Chang, N. L. Seaman, T. T. Warner, and J. M. Fritsch. A two-way interactive nesting procedure with variable terrain resolution. Mon. Wea. Rev., 114:1330-1339, 1986. 